\title{
Prevalence of Carbapenem Resistance and Comparison between Different Phenotypic Methods for Detection of Metallo-B-Lactamases in Gram Negative Non-Fermentative Bacteria in the Acute Wards of a Tertiary Care Centre
}

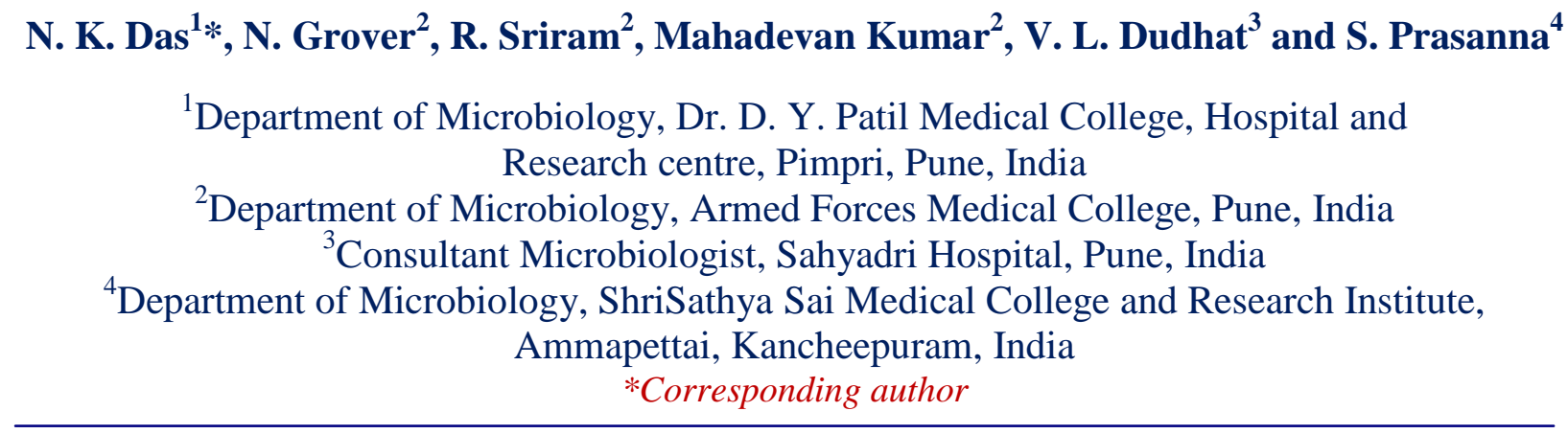

\section{A B S T R A C T}

Keywords

MBL, carbapenemases, Non-fermenters, CDT, DDST, Pseudomonas aeruginosa, Acinetobacter baumannii.

\section{Article Info}

Accepted:

12 April 2016 Available Online: 10 May 2016
Non-fermentative Gram negative bacteria can cause life threatening infections in hospitalised patients. These organisms have high intrinsic antibiotic resistance. Carbapenems are potent agents against these. Production of carbapenemases especially MBLs can jeopardise its use. Study was done to detect the prevalence of carbapenem resistance in NFGNB and to compare the different phenotypic methods for detection of MBLs. Non-duplicate isolates were collected. Isolates were identified by using phenotypic methods and following standard laboratory protocols. Imipenem resistance was detected by using CLSI guidelines. A total of 296 isolates were collected. Acinetobacter baumannii was the predominant isolate (44.59\%) followed by Pseudomonas aeruginosa.71.21\% of A.baumannii and $52.06 \%$ of P.aeruginosa were imipenem resistant. $86.17 \%$ of A.baumannii and $82.53 \%$ of P.aeruginosa were modified Hodge test positive.67.02\% of A.baumannii and $66.66 \%$ of P.aeruginosa were Double disk synergy test positive.76.59\% of A.baumannii and $77.77 \%$ of P.aeruginosa were positive for combined disk test. $74.46 \%$ of A.baumannii and $76.19 \%$ of P.aeruginosa were positive for MBL by E-test. "Almost perfect agreement" was seen between CDT and E-test for A.baumannii whereas "Substantial agreement" was seen for P.aeruginosa. Hence CDT can be used as a cheaper method for phenotypic detection of MBLs.

\section{Introduction}

Non-fermentative Gram negative bacteria (NFGNB) like Pseudomonas aeruginosa, Acinetobacter baumannii, Stenotrophomonas maltophilia and Burkholderia cepacia can cause life threatening infections in hospitalised patients. They also have high intrinsic antibiotic resistance which places the patients at high risk (Hancock, 1998). Carbapenems are potent agents used for 
treatment of serious infections caused by these bacteria. These are preferred because they have a broad spectrum of activity and are resistant to hydrolysis by most $\beta$ lactamases including extended spectrum beta lactamases (ESBLs). There is an increase in consumption in carbapenem antibiotics, especially in hospitals that have a high prevalence of ESBLs. Metallo- $\beta$ lactamases (MBLs) are an important group of carbapenemases and production of MBLs is increasingly being reported in NFGNBs, especially in Pseudomonas aeruginosa. MBLs are Ambler class B enzymes, can hydrolyse carbapenems and are inhibited by metal chelators. Its spectrum is broad but it can't hydrolyse Aztreonam (Franco et al., 2010). Genes of MBL like NDM, VIM, SIM etc. have the ability of horizontal transfer between pathogens in a hospital. PCR is accurate and reliable but its availability in resource limited setting is a constraint. Methods available for MBL detection take advantage of the enzyme's dependence upon $\mathrm{Zn}^{++}$, by using chelating agents like EDTA and 2-mercaptopropionic acid to inhibit its activity (Behera et al., 2008). This study was carried out to detect the presence of MBLs in different imipenem resistant nonfermenters using various phenotypic methods and to compare these methods to ascertain which method can be used reliably in laboratories for fast identification of MBLs.

\section{Materials and Methods}

This study was carried out in the department of microbiology of a tertiary care level hospital in western Maharashtra for 1 year and 7 months. All consecutive, nonduplicate isolates of NFGNB were collected from various clinical samples like blood, sputum, tracheal aspirate, urine, pus etc. from hospitalised patients during the study period. All the isolates were identified taking in to account the colony morphology, pigment production and other phenotypic features. Further biochemical tests like oxidase test, fermentation of sugars, motility, nitrate reduction, decarboxylase tests and glucose oxidative fermentative test (OF test) etc. were done for speciation of the non-fermentative Gram negative bacilli according to standard protocol (Govan, 2011).

\section{Antimicrobial Susceptibility}

The antimicrobial susceptibility of all isolates was done by Kirby- Bauer disk diffusion method according to the CLSI guidelines 2012 (CLSI, 2012). Susceptibility of other antimicrobials was done under standard conditions. Carbapenem susceptibility was seen by using imipenem $(10 \mu \mathrm{g})$ discs.

\section{Modified Hodge Test (MHT)}

All the isolates that were imipenem resistant were subjected to Modified Hodge test (MHT) as described by Lee et al., (2001). In a plate of Mueller Hinton agar an inoculum of 0.5 McFarland of E.coli ATCC 25922 was applied. After waiting for 15 mins, imipenem disc $(10 \mu \mathrm{g})$ was placed onto the inoculated MHA plate in the centre and gently pressed down so as to ensure complete contact with the agar plate. 3-4 colonies of the test isolate were taken with a sterile loop and was streaked on the plate from centre to the periphery of the plate. Positive and negative controls were streaked on the same plate at $90^{\circ}$ angulations from centre to periphery for quality control. Carbapenemase producing isolates were detected, when the test isolate produced the enzyme and allowed growth of the carbapenem susceptible strain (E.coli ATCC 25922) towards the imipenem disc. The result was a characteristic cloverleaf- like 
indentation in case of carbapenemase production. The plates were examined after 16-24 hours of inoculation for a clover-leaf type indentation at the intersection of the test organism and the E.coli ATCC 25922, within the zone of inhibition of the carbapenem susceptibility disc. If a cloverleaf indentation of the E.coli ATCC 25922 growing along the test organism growth streak within the disk diffusion zone was produced, then this isolate was considered as carbapenemase producing (Fig 1). If no growth of the E.coli ATCC 25922 along the test organism growth streak within the disk diffusion zone was observed, then this isolate was considered as noncarbapenemase producing isolate.

Phenotypic Methods for Detection of Metallo- $\beta$-lactamases (MBL)

\section{Imipenem EDTA Double Disc Synergy Test (DDST)}

Double disc synergy test was done as described by Lee et al., (2003). Three to five well isolated colonies of same morphology were selected. A 0.5 McFarland solution was made and with the help of a swab the inoculum was applied to a MHA plate with the help of a rotor. A $10 \mu \mathrm{g}$ imipenem disc was applied to the plate. Another sterile filter paper disc was then applied $10 \mathrm{~mm}$ away to the imipenem disc (edge to edge). $10 \mu \mathrm{l}$ of $0.5 \mathrm{M}$ EDTA solution was then added to the filter paper disc $(750 \mu \mathrm{g})$. The plates were then incubated and the results were interpreted after 16-18 hours. The tests were interpreted as positive if a large synergistic zone was formed in between the discs (Fig 2).

\section{Imipenem-EDTA Disc Method (Combined Disc Method)}

Combined disc test was done as described by Yong et al., (2002). This test was done to confirm the production of MBLs phenotypically. Three to five well isolated colonies of same morphology were selected. A 0.5 McFarland solution was made and with the help of a swab the inoculum was applied to a MHA plate with the help of a rotor. Two imipenem discs $(10 \mu \mathrm{g})$ were applied apart to the plate. To one of the discs $10 \mu \mathrm{l}$ of $0.5 \mathrm{M}$ EDTA solution $(750 \mu \mathrm{g})$ was then added. The plates were then incubated and the results were interpreted after 16-18 hours. The test was interpreted as positive for $\mathrm{MBL}$ if the difference in zones of inhibition of $\geq 7 \mathrm{~mm}$ is produced (Fig 3 ).

\section{Epsilometer Test (E-test)}

E-test was done as described by Lee et al., (2005). This test was done to confirm the production of MBLs phenotypically and to determine the MIC. Three to five well isolated colonies of same morphology were selected. A 0.5 McFarland inoculum was made and applied to a MHA plate. After about fifteen minutes the E-strip (imipenem and imipenem+ EDTA) was applied to the plate at the centre. The results were interpreted after 16-18 hours. A tear drop zone of inhibition was taken as the result. The zone edge intersecting the graded strip was taken as the minimum inhibitory concentration of that isolate. IP and IPI readings were taken respectively for each isolate. MIC ratio of IP/IPI of $\geq 8$ (3 log dilutions) was interpreted as MBL producing. Phantom zone or deformation of the ellipses was also interpreted as positive for MBL regardless of IP/IPI ratio (Fig 4).

\section{Results and Discussion}

A total of 296 samples were collected during the study period. One hundred thirty two isolates were Acinetobacter baumannii, 121 were Pseudomonas aeruginosa and 43 other 
NFGNB like Stenotrophomonas maltophilia, Alkaligenes faecalis, Burkholderia cepacia, Sphingomonas paucimobilis, Achromobacter denitrificans, Pseudomonas fluorescens, Pseudomonas putida and Burkholderia pseudomallei were isolated. Out of these 296 isolates, 187 $(63.1 \%)$ were imipenem resistant. Ninety four out of $132(71.21 \%)$ of A. baumannii, 63 out of 121 (52.06\%) of P.aeruginosa and 30 out of 43 isolates of other non-fermenters were found to be imipenem resistant. MHT was positive in $81(86.17 \%)$ isolates of imipenem resistant A.baumannii (IRAB), 52 $(82.53 \%)$ isolates of imipenem resistant P.aeruginosa (IRPA) and7 (23.33\%) of other imipenem resistant non-fermenters isolates. Overall $140(74.86 \%)$ isolates were positive for MHT.

In DDST, overall 109 (58.28\%) imipenem resistant isolates were found to be DDST positive. Sixty three imipenem resistant A.baumannii isolates (67.02\%), 42 P.aeruginosa (66.66\%) and 4 (13.33\%) isolates of other NFGNB were DDST positive.

By Combined disk test, overall 126 (67.37\%) isolates were positive by CDT for MBL. Among individual isolates 72 of imipenem resistant A.baumannii (76.59\%), $49(77.77 \%)$ of P.aeruginosa and 5 (16.66\%) of other non-fermenters were CDT positive.

By E-test, overall 123 (65.77\%) imipenem resistant isolates were positive for MBL by E-test. $70(74.46 \%)$ isolates of imipenem resistant A.baumannii, 48 (76.19\%) of P.aeruginosa and $5(16.66 \%)$ isolates of other non-fermenters were MBL positive by E-test.

With the global spread and increase in the occurrence of different types of carbapenememase producing strains, their early detection is crucial. This will help significantly in timely implementation of strict infection control practices and treatment with alternative antibiotics. Different molecular techniques are available for detection of genes responsible for carbapenem resistance but they are not available in smaller centres. In these centres phenotypic characterisation can be done with limited resources and they are very sensitive in detection of MBLs etc. (Deshmukh et al., 2011).

A total of 296 samples were collected and analysed during the period from Dec 2012 to Jul 2014. The most common isolate in the present study was Acinetobacter baumannii (44.59\%), followed by Pseudomonas aeruginosa $(40.87 \%)$. Other non-fermenters like Stenotrophomonas maltophilia (6.7\%), Alcaligenes faecalis (2.7\%), Burklolderia cepacia (1.35\%), Sphingomonas paucimobilis (1.35\%), Achromobacter denitrificans (0.67\%), Pseudomonas fluorescens (0.67\%), Pseudomonas putida $(0.67 \%)$ and Burkholderia pseudomallei $(0.33 \%)$ were present in decreasing order (Fig 5). Joseph et al., and Irfan et al., also had A.baumanniias their predominant isolate among non-fermenters (Joseph et al., 2011; Irfan et al., 2008). However Kalidaset al., showed higher prevalence of P.aeruginosa (50.24\%) among the non-fermenters from a study in Kolkata (Rit et al., 2013).

Imipenem resistance was seen in 187 isolates $(63.1 \%)$ by Kirby-Bauer disk diffusion method. There are very few literatures from India and they mention prevalence of carbapenem resistance in nonfermenters varying from 36 to $90 \%$ (Goel et al., 2011). Out of these 187 imipenem resistant isolates, $50.26 \%$ isolates were of A.baumannii, $33.68 \%$ of P.aeruginosa and $16.04 \%$ of isolates were of other NFGNB. 
Amudhanet al., found that $64.08 \%$ and $34.07 \%$ of his imipenem resistant NFGNB isolates to be due to A.baumannii and P.aeruginosa respectively (Amudhan et al., 2012). Our study also has A.baumannii as predominant organism having imipenem resistance among all non-fermenters.

Isolate wise distribution showed that a total of 132 isolates collected during the study period were of A.baumannii. $71.21 \%$ of A.baumannii isolates were imipenem resistant. Study by Khajuria et al., reported a prevalence of imipenem resistance in A.baumannii from a centre in western Maharashtra to be at $42.11 \%$ and Shivaprasad et al., have reported prevalence of carbapenem resistance in A.baumannii at $50.59 \%$ (2014). Baran et al., found the prevalence of IRAB to be at $53.7 \%$ from a hospital in Turkey (2008). Kalidas et al., however found IRAB to be at a lower level at $10 \%$. A total of 121 P.aeruginosa were collected during the study period. Out of these $52.06 \%$ were imipenem resistant. Bhalerao et al., have reported imipenem resistance in P.aeruginosa at $67.5 \%$, Behera et al., showed a prevalence of $69 \%$, Onguru et al., had a prevalence of $44.1 \%$, Singh et $a l$. , had a prevalence of $21 \%$ and Carmeliet al., had a prevalence of $13 \%$ and Lin et al., had a prevalenceof $10.2 \%$ (Onguru et al., 2008; Bhalerao et al., 2010; Carmeli et al., 1999; Singh et al., 2009; Lin et al., 2014). Kalidaset al., in his study from Eastern India reported lower resistance to imipenem (9\%) in P.aeruginosa.

All imipenem resistant isolates were screened for production of carbapenemases by MHT. $74.86 \%$ of all imipenem resistant isolates were MHT positive. Amudhan et al., found $94.4 \%$ of imipenem resistant isolates to be MHT positive in NFGNB from a study in south India. In present study $86.17 \%$ of imipenem resistant A.baumannii isolates were MHT positive. Khajuria et al., reported that $60 \%$ of imipenem resistant A.baumannii was MHT positive and Amudhan et al., reported $97.41 \%$ positivity in MHT. $82.53 \%$ of imipenem resistant isolates of P.aeruginosa were MHT positive. Singh et al., had $71 \%$ of their IRPA as MHT positive. In isolates which were negative for MHT other mechanisms of carbapenem resistance like efflux pump activation or closure of ion channels may responsible. Only $23.33 \%$ of other nonfermenters were MHT positive. This highlights that in other non-fermenters, carbapenemase production may not be a predominant mechanism of imipenem resistance.

All imipenem resistant isolates were screened for production of metallo- $\beta$ lactamases (MBL) by doing double disk synergy test (DDST), combined disk test (CDT) and E-test (MBL and MIC determination) (Fig 6, Table 2). 58.28\% of all isolates were MBL positive by DDST whereas $67.37 \%$ were MBL positive by CDT and $65.77 \%$ were found to be MBL positive by E-test method. Amudhan et al., found that $80.4 \%$ of his imipenem resistant isolates were positive for MBL screening which is higher than the present study (15). Farzana R had $68.5 \%$ positivity for DDST and $85.71 \%$ for CDT in imipenem resistant isolates overall (Farzana et al., 2013). The finding is matching ours in the sense that CDT is more sensitive than DDST. 67.02\% of A.baumannii was positive by DDST, $76.59 \%$ by CDT, and $74.46 \%$ by E-test. Khajuria et al., reported DDST, CDT, E-test positivity in $57.41 \%, 47.09 \%$ and $67.74 \%$ of imipenem resistant isolates of A.baumannii respectively. Shivaprasad et al., reported $67.05 \%, 81.8 \%$ and $100 \%$ sensitivity in detecting MBLs by DDST, CDT and E-test respectively. Overall $82.97 \%$ isolates of imipenem resistant A.baumannii were 
positive for MBL by all phenotypic methods. DDST was positive for 5 isolates which were negative for both CDT and Etest and E-test was positive for an isolate which was negative by CDT and DDST. None of the MHT negative isolates were found to be MBL screen positive.

Table.1 Comparison of DDST, CDT, E-test

\begin{tabular}{|l|l|l|l|}
\hline Test & A.baumannii & P.aeruginosa & Others \\
\hline DDST & $63(67.02 \%)$ & $42(66.66 \%)$ & $4(13.33 \%)$ \\
\hline CDT & $72(76.59 \%)$ & $49(77.77 \%)$ & $5(16.66 \%)$ \\
\hline E-test & $70(74.46 \%)$ & $48(76.19 \%)$ & $5(16.66 \%)$ \\
\hline TOTAL & $78(82.97 \%)$ & $51(80.95 \%)$ & $6(20.00 \%)$ \\
\hline
\end{tabular}

Table.2 Comparison of Different Phenotypic Tests for MBL with E-test

\begin{tabular}{|l|l|l|}
\hline Comparison of Tests & k value (95\% C.I) & Interpretation \\
\hline $\begin{array}{l}\text { DDSTand E-test } \\
(\text { A.baumannii) }\end{array}$ & $0.464(0.271-0.657)$ & Moderate agreement \\
\hline $\begin{array}{l}\text { CDT and E-test } \\
(\text { A.baumannii })\end{array}$ & $0.885(0.775-0.995)$ & Almost perfect agreement \\
\hline $\begin{array}{l}\text { DDST and E-test } \\
(\text { P.aeruginosa })\end{array}$ & $0.538(0.314-0.763)$ & Moderate agreement \\
\hline $\begin{array}{l}\text { CDT and E-test } \\
(\text { P.aeruginosa })\end{array}$ & $0.776(0.589-0.963)$ & Substantial agreement \\
\hline
\end{tabular}

Fig.1 MHT Test for Detection of Carbapenemases (PC-Positive Control, NC- Negative Control, PT- Positive Test, NT- Negative Test)

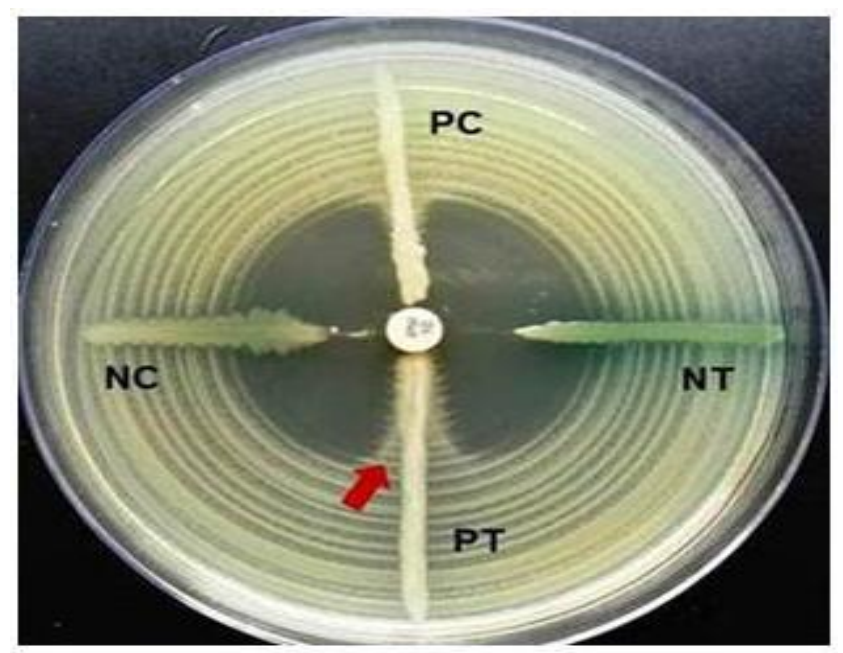


Fig.2 Imipenem-EDTA DDST Test for MBL Screening; Zone of Accentuation between Imipenem and EDTA

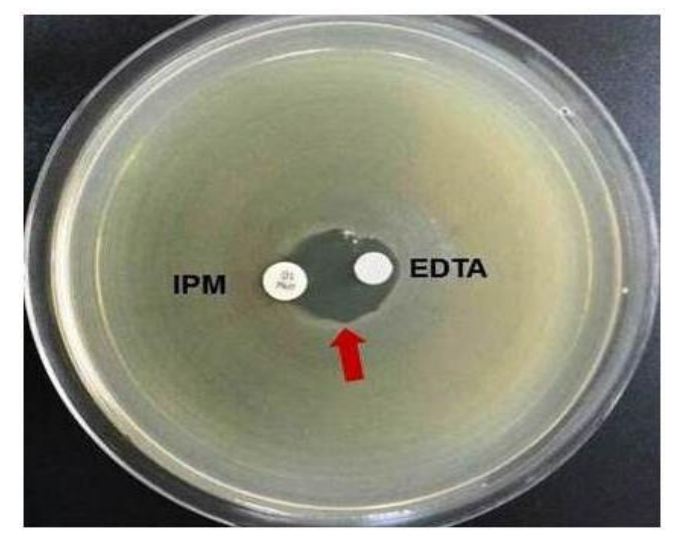

Fig.3 Imipenem EDTA Combined Disc Test (CDT) for MBL Screening; Imipenem and EDTA Zone Size $>7 \mathrm{~mm}$ than IPM Zone Size

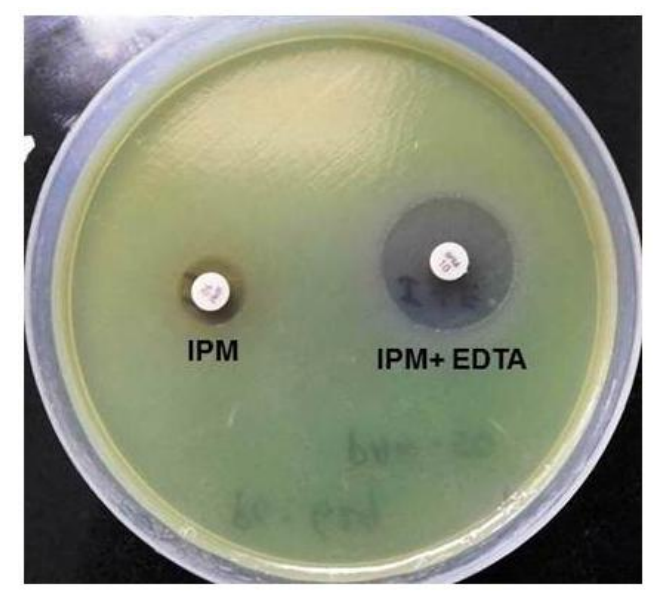

Fig.4 MBL Test for Pseudomonas aeruginosa by E-test; MIC> 256 and MBL Positive

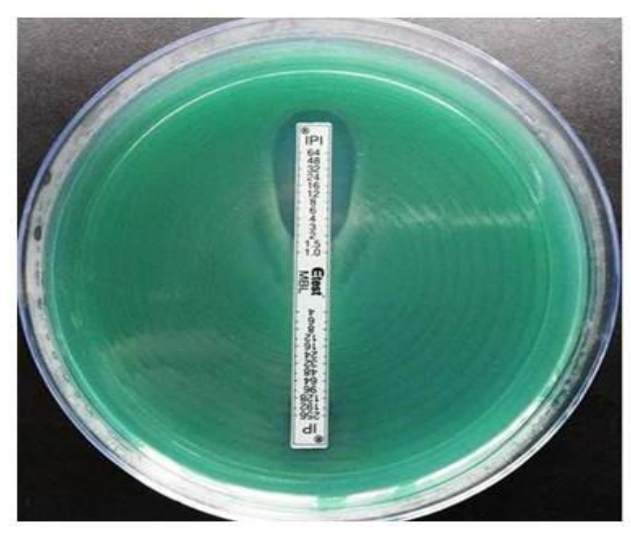


Fig.5 Species Wise Distribution in all Samples

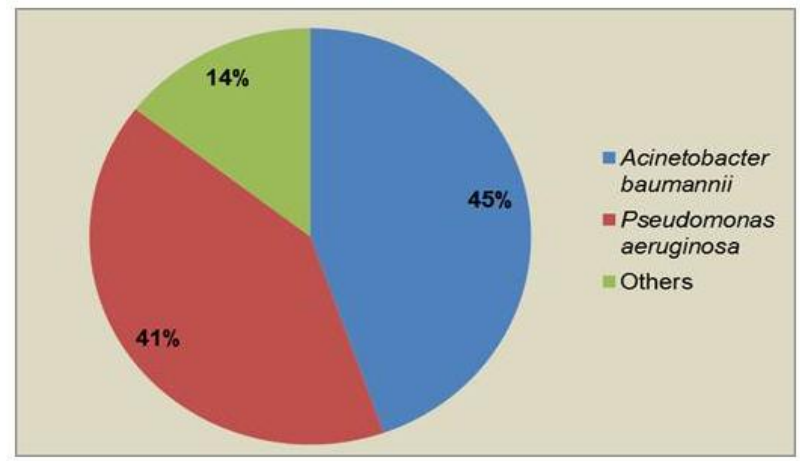

Fig.6 Comparison between Different Phenotypic Tests

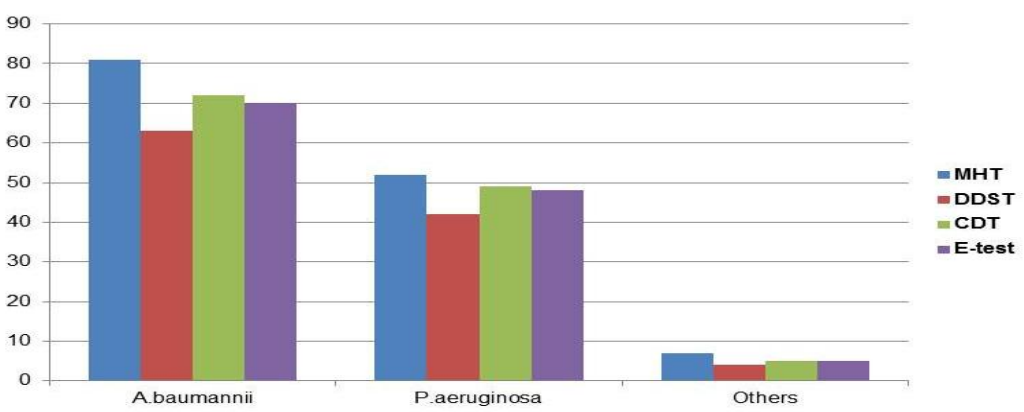

In P.aeruginosa $66.66 \%$ of isolates were positive for MBL by DDST, $77.77 \%$ by CDT and $76.19 \%$ by E-test. Behera et al., reported that $85.71 \%$ and $64.28 \%$ of imipenem resistant isolates were positive for screening by CDT and DDST respectively which matches our study in the sense that CDT proved to be more sensitive for screening for MBL than DDST. They also reported 100\% sensitivity by E-test for MBL which is in contrast to the present study in which we are reporting $76.19 \%$ IRPA by Etest. This discrepancy may be because of low number of isolates tested by them. However in our study E-test was able to pick up some MBLs which were negative by CDT and DDST, which matches with that of Behera et al., Bhalerao et al., reported DDST to screen $50 \%$ of MBLs and CDT picked up 66.7\% of MBLs which is lower than what we found in present study. The possible explanation for this may be the highly subjective interpretation of results in DDST or there may be low incidence of MBLs in their setting. In all $80.95 \%$ of isolates showed MBL production by all three screening methods. Varying prevalence of MBL is seen worldwide, 39\% in Italy and $44 \%$ in Brazil. All isolates for MBL screen were positive by CDT except two, which were positive by E-test only. In rest of imipenem resistant isolates other mechanisms may be responsible for imipenem resistance. No MHT negative isolate was positive for any MBL screening test positive.

In other non-fermenters $13.33 \%$ were MBL 
producing by DDST, $16.66 \%$ by CDT and $16.66 \%$ by E-test. In all $20 \%$ of isolates were positive for MBL by all screening tests. This suggests that mechanisms other than MBL may be responsible for carbapenem resistance in them.

DDST and CDT were compared with E-test for MBL detection using kappa co-efficient (k) (25). According to Khosravi et al., E-test is $100 \%$ sensitive and specific in detection of MBLs in IRPA (Khosravi et al., 2012). So in the present study we did comparison to see the agreement between CDT and E-test and DDST and E-test. We found moderate agreement between DDST and E-test in both A.baumannii $(\mathrm{k}=0.464)$ andP.aeruginosa $(\mathrm{k}=0.538) \quad$ whereas absolutely perfect agreement was seen between CDT and Etest in A.baumannii $(\mathrm{k}=0.885)$ and substantial agreement $(\mathrm{k}=0.776)$ was seen between CDT and E-test in P.aeruginosa (Table 2). Based on this finding we can safely say that CDT can be used as a suitable alternative for E-test as it's cost effective and can be relied upon for detection of MBLs in routine clinical laboratories. Behera et al., also recommended the use of CDT for the phenotypic detection of MBL in laboratories routinely.

In conclusion, Carbapenem resistance is emerging as a major problem in treatment of patients in many clinical settings around the globe. The problem is getting compounded due to the rapid spread of strains harbouring these resistant genes. Carbapenem is being used as a broad spectrum antibiotic especially in critically ill patients. Only few reserve drugs are present for carbapenem resistant isolates but it is associated with many side effects. Multiple mechanisms of carbapenem resistance are present in NFGNB. The carbapenem resistance mechanism in NFGNB in our hospital setting is mostly because of metallo- $\beta$ lactamases and other carbapenemases enzyme. Just by doing a few simple phenotypic tests like MHT, CDT the presence of carbapenemases and MBL can be detected. Since there are limited treatment options against these, continuous vigilance, early identification and treatment is very important to prevent further spread. Constant and regular monitoring of the incidence of such organisms in various critical areas of the hospital like ICU and acute medical units, prompt recognition of potential areas of colonisation and getting rid of them is the only important preventive strategy. To keep this problem in check, simple infection control measures like proper hand washing, adherence to infection control guidelines and antibiotic stewardship must be followed and time to time revision must be done.

\section{References}

Hancock, R.E. 1998. Resistance mechanisms in Pseudomonas aeruginosa and other nonfermentative gram negative bacteria. Clin. Infect. Dis., 27: S93-9.

Franco, M.R.G., Caiaffa-Filho, H.H., Burattini, M.N. et al. 2010. Metallobeta-lactamases among imipenemresistant Pseudomonas aeruginosa in a Brazilian university hospital. Clinics, 65(9): 825-829.

Behera, B., Mathur, P., Das, A., et al. 2008. Indian J. Med. Microbiol., 26(3): 233-7.

Govan, J.R.W. 2011. Pseudomonas, Stenotrophomonas, Burkholderia. In: Collee JG, Fraser AG, Marmion BP, Simmons A, editors. Mackie \& McCartney Practical Medical Microbiology. $14^{\text {th }}$ ed. India, Churchilll Livingstone. 
CLSI. 2012. Performance standards for antimicrobial susceptibility testing; twenty-second informational supplement. CLSI document M100S22. Wayne, PA. Clinical and Laboratory Standards Institute.

Lee, K., Chong, Y., Shin, H.B., Kim, Y.A., Yong, D., Yum, J.H. 2001. Modified Hodge and EDTA-disk synergy tests to screen metallo- $\beta$-lactamaseproducing strains of Pseudomonas and Acinetobacter species. Clin. Microb. Biol. Infect., 7(2): 88-102.

Lee, K., Lim, Y.S., Yong, D., Yum, J.H., Chong, Y. 2003. Evaluation of the Hodge Test and the ImipenemEDTA Double-Disk Synergy Test for Differentiating Metallo- $\beta$ Lactamase-Producing Isolates of Pseudomonas spp. and Acinetobacter spp. J. Clin. Microbiol., 4623-9.

Yong, D., Lee, K., Yum, J.H., Shin, H.B., Rossolini, G.M., Chong, Y. 2002. Imipenem-EDTA Disk Method for Differentiation of Metallo- $\beta$ Lactamase-Producing Clinical Isolates of Pseudomonas spp. and Acinetobacter spp. J. Clin. Microbiol., 40(10): 3798-801.

Lee, K., Yong, D., Yum, J.H., Lim, Y.S., Bolmstrom, A., Qwarnstrom, A., et al. 2005. Evaluation of Etest MBL for detection of $b a_{I M P-1}$ and $b l a_{V I M-2}$ Allele-positive clinical isolates of Pseudomonas spp. and Acinetobacter spp. J. Clin. Microbiol., 43(2): 9424.

Deshmukh, D.G., Damle, A.S., Bajaj, J.K., Bhakre, J.B., Patwardhan, N.S. 2011. Metallo- $\beta$-lactamase producing clinical isolates from patients of a tertiary care hospital. J. Lab. Physicians, 3(2): 93-7.

Joseph, N.M., Sistla, S., Dutta, T.K., Badhe, A.S., Rasitha, D., Parija, S.C. 2011. Reliability of Kirby-Bauer disk diffusion method for detecting meropenem resistance among nonfermenting gram negative bacilli. Indian J. Pathol. Microbiol., 54(3): 556-60.

Irfan, S., Zafar, A., Guhar, D., Ahsan, T., Hasan, R. 2008. Metallo- $\beta$ lactamase-producing clinical isolates of Acinetobacter species and Pseudomonas aeruginosa from intensive care units of a tertiary care hospital. Indian J. Med. Microbiol., 26(3): 243-5.

Rit, K., Nag, F., Raj, H.J., Maity, P.K. 2013. Prevalence and susceptibility profiles of non-fermentative gram-negative bacilli infection in a tertiary care hospital of Eastern India. Indian $J$. Clin. Practice, 24(5): 451-5.

Goel, N., Wattal, C., Oberoi, J.K., Raveendran, R., Datta, S., Prasad, K.J. 2011. Trend analysis of antimicrobial consumption and development of resistance in nonfermenters in a tertiary care hospital in Delhi, India. J. Antimicrob. Chemother., 60(7): 1625-30.

Amudhan, M.S., Sekar, U., Kamalanathan, A., Balaraman, S. bla $a_{I M P}$ and bla $a_{V I M}$ mediated carbapenem resistance in Pseudomonas and Acinetobacter species in India. J. Infect. Dev. Ctries., 6(11): 757-62.

Khajuria, A., Praharaj, A.K., Kumar, M., Grover, N. 2014. Molecular characterisation of carbapenem resistant isolates of Acinetobacter baumannii in an intensive care unit of a tertiary care centre. J. Clin. Diag. Res., 8(5): 38-40.

Shivaprasad, A., Antony, B., Shenoy, P. 2014. MBL \& Carbapenemase Production in A.baumannii. J. Clin. Diag. Res., 8(5): 5-8. 
Baran, G., Erbay, A., Bodur, H., Onguru, P., Akinci, E., Balaban, N., et al. 2008. Int. J. Infect. Dis., 12: 16-21.

Onguru, P., Erbay, A., Bodur, H., Baran, G., Akinci, E., Balaban, N., et al. 2008. Imipenem resistant Pseudomonas aeruginosa: risk factors. J. Korean Med. Sci., 23: 982-7.

Bhalerao, D.S., Roushani, S., Kinikar, A.G., Akhter, I. 2010. Study of metallobeta lactamase producing Pseudomonas aeruginosa in Pravara rural hospital. Pravara Med. Rev., 2(3): 16-9.

Carmeli, Y., Troillet, N., Eliopoulos, G.M., Samore, M.H. 1999. Antimicrob. Agents Chemother., 43(6): 1379-82.

Singh, S.P., Shariff, M., Barua, T., Thukral, S.S. 2009. Comparative valuation of phenotypic tests for identification of metallo- $\beta$-lactamases producing clinical isolates of Pseudomonas aeruginosa. Indian J. Med. Res., 713-5.
Lin, K.Y., Lauderdale, T.L., Wang, J.T., Chang, S.C. 2014. Carbapenem resistant Pseudomonas aeruginosa in Taiwan: Prevalence, risk factors, and impact on outcome of infections. $J$. Microbiol. Immunol. Infect., 14: 8-5.

Farzana, R., Shamsuzzaman, S.M., Mamun, K.Z. 2013. Isolation and molecular characterisation of New Delhi metallo- $\beta$-lactamase-1 producing superbug in Bangladesh. Emerg. Problems in Infect. Dis., 7(3): 161-8.

Viera, A.J., Garrett, J.M. 2005. Understanding interobserver agreement: the kappa statistic. Fam. Med., 37(5): 360-3.

Khosravi, Y., Loke, M.F., Chua, E.G., Tay, S.T., Vadivelu, J. 2012. Phenotypic detection of metallo- $\beta$-lactamase in imipenem resistant Pseudomonas aeruginosa. The Scientific World J., 1-7.

\section{How to cite this article:}

Das, N.K., N. Grover, R. Sriram, Mahadevan Kumar, V.L. Dudhat and Prasanna, S. 2016. Prevalence of Carbapenem Resistance and Comparison between Different Phenotypic Methods for Detection of Metallo-B-Lactamases in Gram Negative Non-Fermentative Bacteria in the Acute Wards of a Tertiary Care Centre.Int.J.Curr.Microbiol.App.Sci. 5(5): 109-119. doi: http://dx.doi.org/10.20546/ijcmas.2016.505.012 